\title{
A MILLIMETER WAVE TECHNIQUE FOR MEASURING ICE THICKNESS ON THE SPACE SHUTTLE'S EXTERNAL TANK
}

\author{
John E. Kendra, ${ }^{1}$ Fawwaz T. Ulaby, ${ }^{1}$ and Steve $\mathbf{W u}{ }^{2}$ \\ 'Radiation Laboratory, Electrical Engineering, Computer Science \\ University of Michigan \\ Ann Arbor, Michigan 48109-2122 \\ ${ }^{2} N A S A$ Stennis Space Center \\ NSTL \\ Missisippi 39529
}

Received September 27, 1991

\begin{abstract}
The external fueltank of the Space Shuttle contains extremely lowtemperature propellents. A layer of material known as SOFI (SprayOn Foam Insulation) covering the outside of the fueltank provides thermal insulation between the aluminum surface of the fueltank and the ambient air. In spite of this insulation, under certain conditions ice formation will occur on the surface of the SOFI. Ice on the external fueltank can be detrimental to the launch and it is important to detect its presence and measure its thickness. This paper describes the design of a millimeter-wave radiometer technique developed for this purpose. The design is based on model calculations and measurements of the emission properties of a panel from the external fueltank, performed at 35, 94 and $140 \mathrm{GHz}$. Two sets of measurements were performed, one for the unmodified rough-surface SOFI panel and another for a


panel whose surface was sanded down to produce a smooth surface interface with the ice cover. The latter was used to evaluate the results of radiative transfer calculations which are much easier to perform for multilayer structures with plane boundaries. We present experimental evidence demonstrating that the technique developed can accurately predict ice thickness in the case of the smooth-surface SOFI panel. For the original (rough-surface) panel, the emission levels observed were considerably higher than predicted by the model. Both cases however exhibited comparable sensitivities to ice thickness ( $\sim 1 \mathrm{~K} / \mathrm{mm}$ at 35 $\mathrm{GHz}, 4 \mathrm{~K} / \mathrm{mm}$ at $94 \mathrm{GHz}$, and $5 \mathrm{~K} / \mathrm{mm}$ at $140 \mathrm{GHz}$ ).

\section{Introduction}

The external fuel tank of the space shuttle contains large reservoirs of liquid oxygen and liquid hydrogen. As these propellants exist at extremely low temperatures, the outside surface of the fuel tank is covered with a layer of insulation material called SOFI (Spray-On Foam Insulation). Despite this measure, under certain conditions ice will form on the SOFI surface due to condensation and freezing of atmospheric water vapor. The presence of ice on the external tank can be detrimental to the launch and it is important to detect its presence and measure its thickness. In this study, we investigate the feasibility of using a millimeter-wave imaging radiometer for this purpose.

The proposed system is illustrated in Figures 1 and 2. The imaging radiometers are placed several hundred meters away from the launch pad, and their beams image the external tank over a narrow angular range centered around $45^{\circ}$. The inset of Figure 1 shows additional detail, along with typical parameters, for the case in which ice is present.

Figure 2 shows a conceptual design of the imaging radiometer. The radiometer antenna consists of a cylindrical reflector fed by a monolithic array of mixer elements. The array will produce continuous beams on the fuel tank surface. By rotating the array about its own axis, the surface will be imaged in the vertical dimension, thereby producing a two-dimensional image of the surface.

Before proceeding further, we now list two modifications we have imposed to allow us to examine the antenna temperature measured by the radiometer in terms of exact radiative transfer models:

1. The original SOFI material has a rough surface. In this study, 


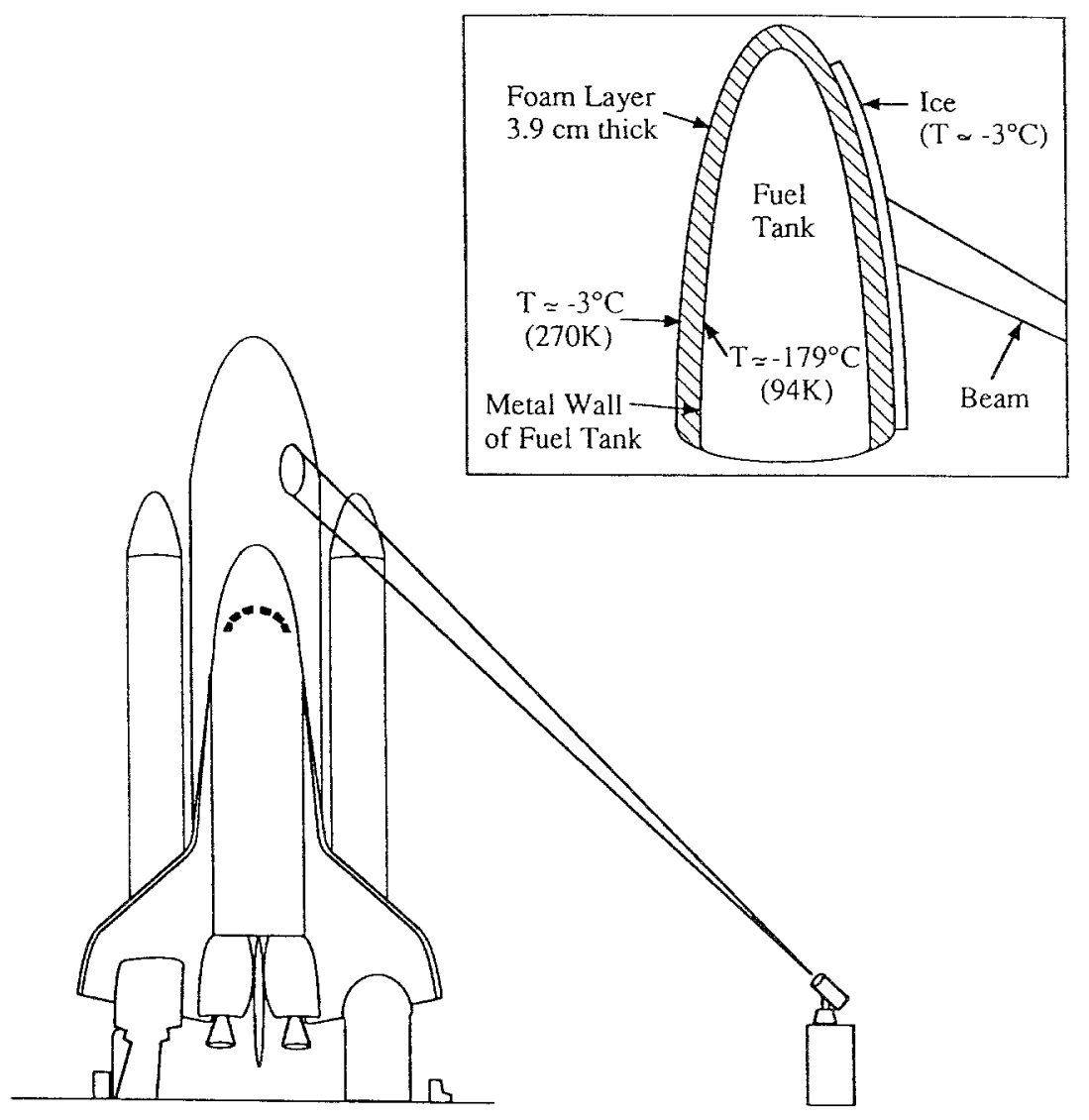

Figure 1: Millimeter-wave imaging system. Inset shows detail of case where ice is present. 


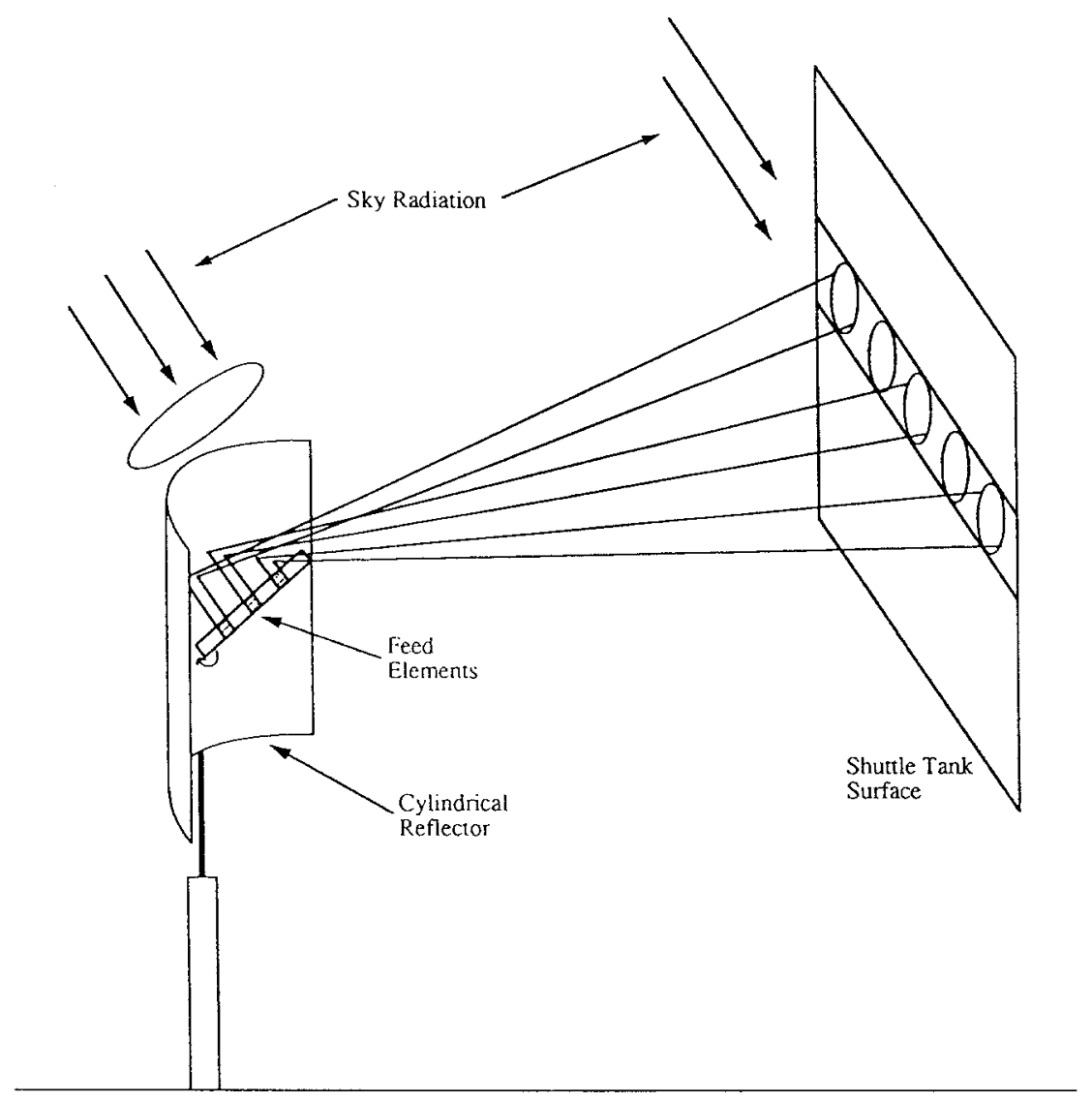

Figure 2: A conceptual configuration for an imaging millimeter-wave radiometer that uses a linear antenna array to feed a cylindrical reflector. As the array rotates about its own axis, the beams scan the shuttle tank surface along the vertical direction. At its end position, the array views the sky radiation through a focusing lens. 
though we will briefly discuss the emission behavior of the rough panel when covered with an ice layer, the subject of the bulk of our work, and the basis for our models, is a SOFI panel whose surface has been sanded down to a smooth surface.

2. Initially, we treat the system (ice over SOFI panel) as if it were isothermal. From Figure 1 it is evident that in reality there is a temperature gradient between the metal wall of the tank and the ambient air. Later in this paper, we will show how the technique developed for the isothermal system may be extended to the case of a temperature gradient.

The first simplification allows us to express the antenna temperature $T_{A}$ as [1]:

$$
T_{A}=T_{B}+R T_{S K Y},
$$

where $T_{B}$ is the brightness temperature of the fuel tank surface, $R$ is its effective reflectivity, and $T_{S K Y}$ is the sky radiation incident upon the fuel tank in the specular direction. Incorporating the second assumption above allows further simplification to:

$$
T_{A}=e T_{\text {phys }}+(1-e) T_{S K Y}
$$

where $e$ is the emissivity and $T_{\text {phys }}$ is the physical (uniform) temperature of the medium. The combination of the isothermal and smooth-surface assumptions allowed us to use the relation

$$
e(\theta, \phi)=1-R(\theta, \phi) .
$$

Rearranging (2) allows us to express the emissivity in terms of three measurable quantities:

$$
e=\frac{T_{A}-T_{S K Y}}{T_{p h y s}-T_{S K Y}} .
$$

Note that in Figure 2, after the feed array completes its scan of the antenna reflector (as it rotates about its own axis), it will observe the sky radiation directly through a focusing lens. The observation direction will be the same as that of the sky radiation incident upon the fuel tank surface. Hence, $T_{S K Y}$ will be measured directly. 
In our investigation, we will determine how emissivity, as determined in (3), can be related to ice thickness, thereby providing a means to measure the latter, in the isothermal case. Finally, we will demonstrate how this same procedure can be applied to the case of a temperature gradient, which more closely resembles the actual situation with the fuel tank. For this case, where (2) is no longer completely valid, we introduce the concept of an effective emissivity, $e_{e f f}$.

\section{Characterization of SOFI Material}

In order to model the emissivity of the system consisting of an ice layer over the fuel tank surface, it is necessary to know certain electromagnetic properties of each of the components that comprise the system. In this section, we analyze the emissive characteristics of the fuel tank surface by itself, without ice.

The wall of the fuel tank consists of metal covered by a layer of SOFI, a foam-like material, having a thickness of approximately 3.9 $\mathrm{cm}$. According to $\mathrm{Wu}[2]$, the temperature at the metal-SOFI interface (Figure 3 ) is on the order of $94 \mathrm{~K}$ (or $-179^{\circ} \mathrm{C}$ ) and the temperature in the ice layer is a few degrees below the freezing temperature of water; i.e., typically $270 \mathrm{~K}$ ( or $-3^{\circ} \mathrm{C}$ ). The first step of this investigation is to determine whether this material behaves (electromagnetically) like a homogeneous layer or an inhomogeneous layer. If the material is homogeneous in behavior, coherent emission models are applicable, whereas if it is inhomogeneous, incoherent emission models should be used instead. The appropriate model can then be used in determining the relative dielectric constant of the SOFI material. The behavior of this fuel tank wall material is examined with respect to emissivity as a function of incidence angle. Figure 4 shows the arrangements used to measure $T_{S K Y}$ and $T_{A}$, from which $e(\theta)$ is computed according to (3). For each angle $\theta$ (Figure 4 ), $T_{S K Y}$ was measured by observing a flat metal panel, and then $T_{A}$ was measured after replacing the metal panel with the SOFI panel. The measurements were conducted at 35, 94, and $140 \mathrm{GHz}$, for both horizontal and vertical polarizations. All three radiometers used $15-\mathrm{cm}$ diameter corrugated-lens horn antennas with beamwidths of $3.2^{\circ}$ at $35 \mathrm{GHz}, 1.4^{\circ}$ at $94 \mathrm{GHz}$, and $0.9^{\circ}$ at $140 \mathrm{GHz}$. Radiometer calibration was achieved by placing each of two specially 


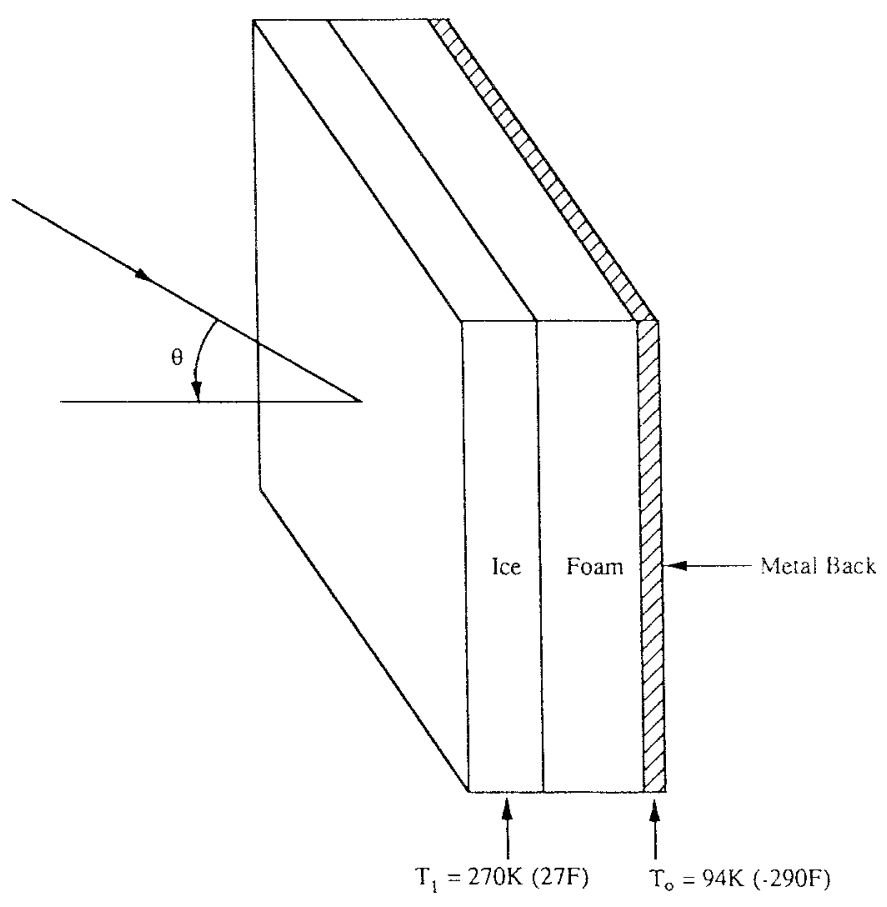

Figure 3: The tank surface is covered with a foam layer, approximately $3.9 \mathrm{~cm}$ thick, on the surface of which ice can form by condensation and freezing. The foam layer, called SOFI (Spray On Foam Insulation), acts as a thermal insulator between the low temperature metal surface and the outside environment. 


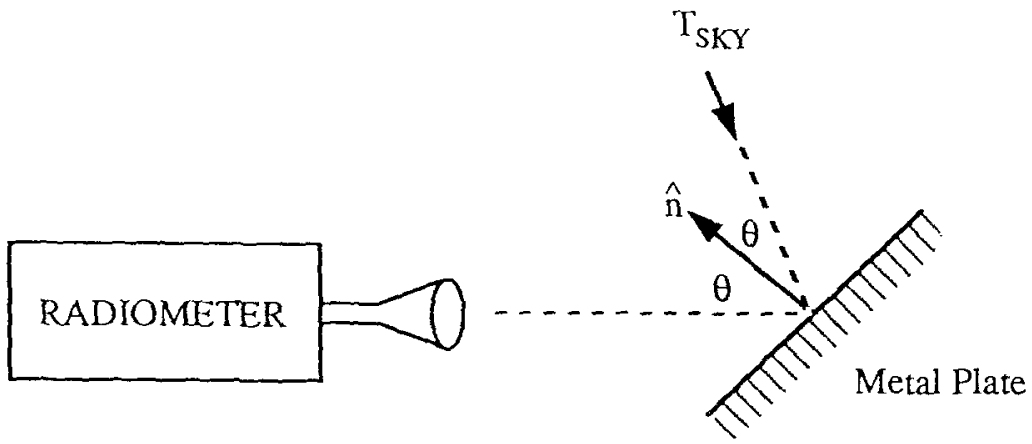

(a) Measurement of $\mathrm{T}_{\mathrm{SKY}}$.
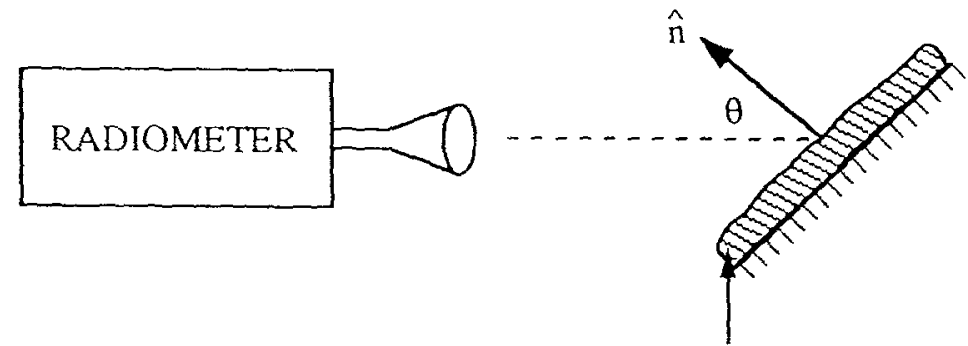

SOFI Panel

(b) Measurement of emission by SOFI Panel.

Figure 4: The arrangements used for measuring (a) $T_{S K Y}$ and (b) the antenna temperature due to emission by the SOFI panel. The angle $\theta$ can be adjusted to cover the range from $20^{\circ}$ to $70^{\circ}$. 
constructed panels of absorbing material (with emissivity $\simeq 1$ ) immediately in front of the antenna and then measuring the output voltage. The first panel is at the ambient air temperature and the second panel is at a temperature of $77 \mathrm{~K}$, achieved by pouring liquid nitrogen into the absorbing material through narrow holes cut into the material. The physical temperatures are measured by thermistors imbedded inside the material.

The measurement procedure was carried out on both the original rough-surface SOFI panel and the modified, smooth-surface version of the SOFI panel. The results of the measurements of the original (rough) SOFI panel are shown in Figures 5 and 6 . Figure 5 shows the measured angular response of the emissivity $e$ at 35 and $140 \mathrm{GHz}$ for the original rough SOFI panel. Figure 6 shows the same information at $94 \mathrm{GHz}$, for both the rough surface panel and the smooth surface SOFI panel. We observe that except for some very small oscillations, the emissivity increases monotonically with increasing incidence angle at all three frequencies for both polarizations.

The results from the smooth SOFI panel, shown in Figure 6(b) (94 $\mathrm{GHz}$ only), indicate that except for an overall reduction in emissivity, due to the thickness of the layer having been reduced by the sanding process to $2.7 \mathrm{~cm}$, the data resembles that of the rough panel.

Both test panels, rough and smooth, consist of a low-loss dielectric slab covering a metal plate. For a homogeneous slab with plane boundaries, theory suggests that we should observe large oscillations as a function of incidence angle. The absence of such oscillations in the observed data is indicative that phase coherence of the multiple reflections between the lower and upper boundaries of the dielectric slab is not preserved, which can be attributed to lack of dielectric homogeneity of the slab material at the scale of the wavelength under consideration and/or to the roughness of the upper boundary. When the rough upper boundary is removed, as in the case of the smooth panel, the essential emission characteristics of the panel are unchanged. Evidently, the rough surface is not an important factor in the emission of the SOFI panel itself. From these results on both test panels, it is clear that the emission by the SOFI panel should be modeled using an incoherent radiative transfer approach.

For an inhomogeneous slab of thickness $d$ and a relative dielectric constant $\epsilon=\epsilon^{\prime}-j \epsilon^{\prime \prime}$ supported by a metal back surface, radiative 
(a) Rough-Surface SOFI, $35 \mathrm{GHz}$

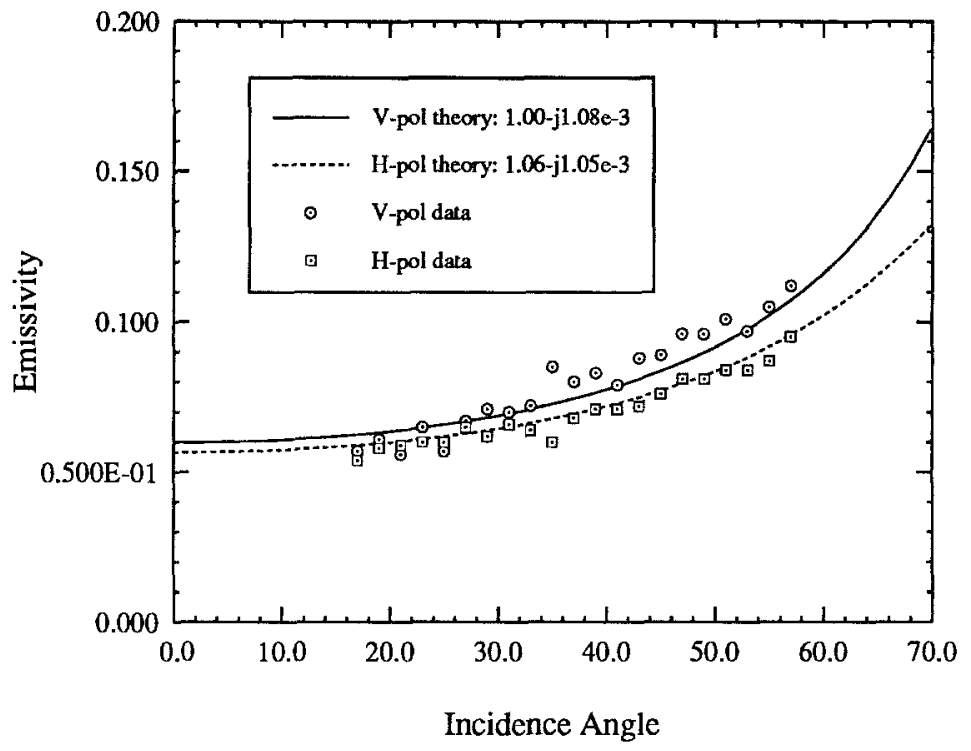

(c) Rough-Surface SOFI, $140 \mathrm{GHz}$

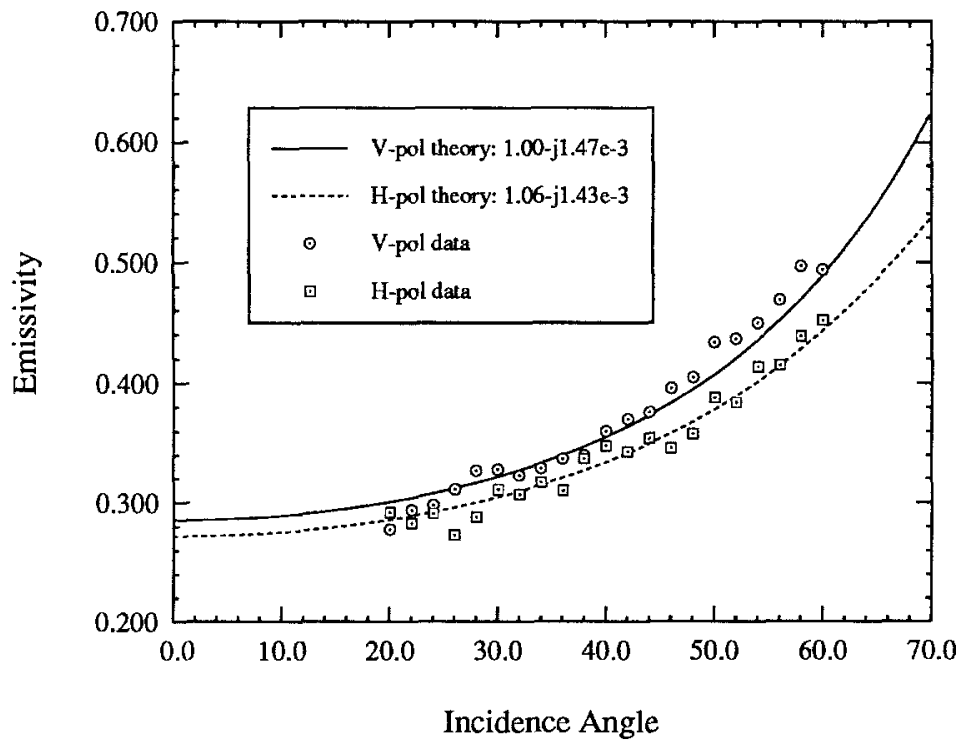

Figure 5: Measured and calculated emissivity: (a) $35 \mathrm{GHz}$, rough surface; (b) $140 \mathrm{GHz}$, rough surface. 
(b) Rough-Surface SOFI, $94 \mathrm{GHz}$

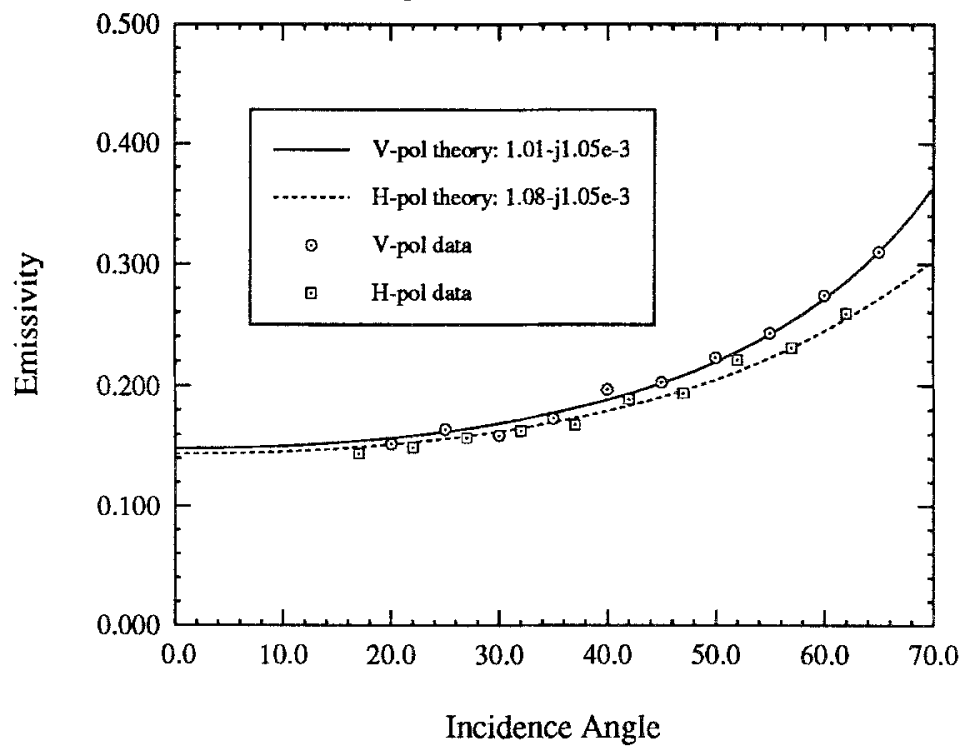

(d) Smooth-Surface SOFI, $94 \mathrm{GHz}$

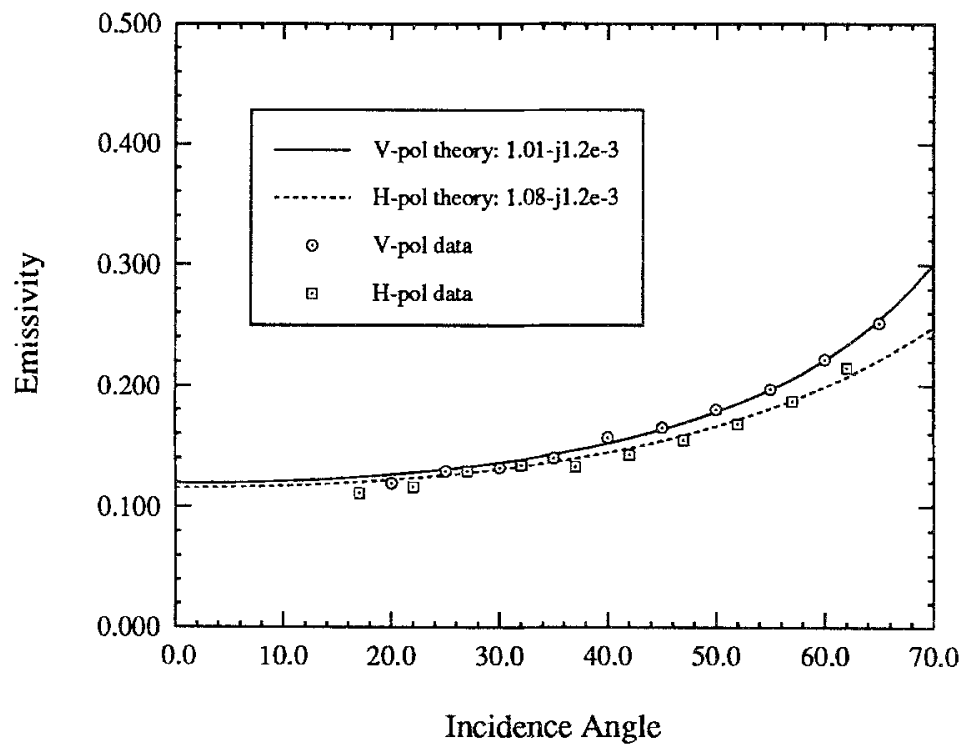

Figure 6: Measured and calculated emissivity: (a) $94 \mathrm{GHz}$, rough surface; (b) $94 \mathrm{GHz}$, smooth surface. The rough-surface panel was $3.9-\mathrm{cm}$ thick, compared to $2.7-\mathrm{cm}$ for the smooth-surface panel. 
transfer theory provides the following expression for the emissivity $e_{p}(\theta)$ at incidence angle $\theta$ and antenna polarization $p(v$ or $h)$,

$$
e_{p}(\theta)=\frac{\left(1-\Upsilon^{2}(\theta)\right)(1-R(p, \theta))}{1-\Upsilon^{2}(\theta) R(p, \theta)},
$$

where

$$
\Upsilon^{2}=\exp \left(-2 \kappa d \sec \theta^{\prime}\right),
$$

$\kappa$ is the absorption coefficient of the SOFI material, $\theta^{\prime}=\sin ^{-1}\left(\sin \theta / \sqrt{\epsilon^{\prime}}\right)$ is the refraction angle, and $R(p, \theta)$ is the p-polarized Fresnel reflectivity of the upper surface.

For a low-loss material, $\kappa$ is given by the approximate expression:

$$
\kappa \simeq \frac{2 \pi}{\lambda} \frac{\epsilon^{\prime \prime}}{\sqrt{\epsilon^{\prime}}}
$$

Using a $5-\mathrm{GHz}$ dielectric probe, it was determined that $\epsilon^{\prime}$ of the SOFI material is less than 1.3. For a material with such a relative permittivity, the Fresnel reflectivity at normal incidence is 0.004 . This means that the emissivity, as given by (4), is approximately

$$
e(\theta=0) \simeq 1-\Upsilon^{2}(0) .
$$

By extrapolating the measured data down to $0^{\circ}$, we obtain essentially (to within experimental error) the same value for $v$ and $h$ polarizations, as expected. Upon using these values in (6), we obtain a value for $\Upsilon^{2}$ (0) at each of the three frequencies. This, in turn, specifies the value of the ratio $\epsilon^{\prime \prime} / \sqrt{\epsilon^{\prime}}$ from (5) at each frequency. Finally, with $\epsilon^{\prime}$ treated as a free variable, (4) is used to compute $e_{p}(\theta)$ versus $\theta$ to determine the effective value of $\epsilon^{\prime}$ that provides a good fit to the observed data. The results of this process are shown in Figures 5 and 6 where we observe that the calculated curves are in very good agreement with the data. The values of $\epsilon$ used in the calculations are given in the insets of Figure 5 and 6.

These results raise two questions: a) why is $\epsilon$ slightly different for the two polarizations, and b) why is the value of $\epsilon$ for the smoothsurface panel slightly different from that of the rough-surface panel? The answers to both of these questions lies in the structure of the SOFI material. It is not actually a continuous slab, but has instead four or five layers of identical material, separated by thin darker layers. These layers are evidently produced in the application process. 
In light of the above discussion, we shall treat the experimentally derived values of $\epsilon$ as effective values representing the multilayered inhomogeneous SOFI panel. These values of $\epsilon$ will be used in succeeding sections to calculate the emission behavior of the SOFI panel when covered by a sheet of ice.

\section{Determination of Ice Dielectric Prop- erties}

As discussed earlier, under certain conditions, the presence of a cold SOFI panel surface induces the formation of ice (or frost) through condensation and freezing of water vapor from the ambient environment. This frost layer is likely to be a combination of air and ice particles, which raises two questions: (a) what is an appropriate value of the ice volume fraction that should be used in characterizing the dielectric of the frost layer, and (b) what is the dielectric constant of ice at the frequencies under consideration in this study?

\subsection{Ice Volume Fraction}

Due to the lack of information pertinent to the first question, we should keep the ice volume fraction as an unknown variable for the present. However the form of the computations associated with this parameter is as detailed below.

To compute the relative dielectric constant of a frost layer, $\epsilon_{1}$, we shall assume that the ice particles in the layer are spherical in shape and much smaller than $\lambda$ in size, in which case we can use the mixing model $[1$, Appendix E]

$$
\begin{gathered}
\epsilon_{1}^{\prime}=\frac{1+0.835 v}{1-0.417 v}, \\
\epsilon_{1}^{\prime \prime}=\frac{0.34 v \epsilon_{i}^{\prime \prime}}{(1-0.417 v)^{2}},
\end{gathered}
$$

where $\epsilon_{i}^{\prime \prime}$ is the imaginary part of the relative dielectric constant of pure ice and $v$ is the ice volume fraction. In emission calculations presented later in this report, we shall consider two cases for $v$, namely $v=0.5$, which we shall refer to as frost, and $v=1$ representing pure ice. 


\subsection{Ice Dielectric Properties}

For pure ice, the relative dielectric constant $\epsilon_{i}$ is given by

$$
\epsilon_{i}=\epsilon_{i}^{\prime}-j \epsilon_{i}^{\prime \prime},
$$

with $\epsilon_{i}^{\prime}=3.15$, independent of temperature and frequency at microwave and millimeter-wave frequencies [1, Appendix E]. The imaginary part, $\epsilon_{i}^{\prime \prime}$, on the other hand, exhibits a strong dependance on frequency and a weak dependence on temperature [1].

To determine the dielectric constant of the ice used in this study we used the same technique employed by Mätzler and Wegmüller [3] in an extensive ice study undertaken in 1987. The procedure involves observing, at the Brewster angle $\theta_{B}$, the vertically polarized emission from a layer of ice over a metal surface. The setup is illustrated in Figure 7 . The sky brightness, $T_{S K Y}$, suffers no reflection at the air/ice interface, either entering or exiting the ice layer. Therefore, the effective reflectivity is just the amount by which $T_{S K Y}$ is attenuated by the ice layer. Thus,

$$
R\left(\theta_{B}\right)=1-e\left(\theta_{B}\right)=\Upsilon^{2}\left(\theta_{B}\right),
$$

where $\Upsilon^{2}$ is as given in (4) and $\kappa$ (contained in $\Upsilon$ ) is as given in (5). Since $\epsilon_{i}^{\prime}$ is known, $\epsilon_{i}^{\prime \prime}$ can be calculated directly.

In addition to observing the ice at the Brewster angle, as an additional check, we further measured the emissivity at other incidence angles between $15^{\circ}$ and $70^{\circ}$. This was realized using the same technique described for the SOFI panel. If the value for $\epsilon_{i}^{\prime \prime}$ calculated from the Brewster angle is correct, then the emissivities measured at other incidence angles should be predicted by an emission model utilizing $\epsilon_{i}^{\prime \prime}$.

At $94 \mathrm{GHz}$ the measurement process was performed with two different thicknesses of ice, using vertical polarization only. As shown in Figure 8 , a very good fit was achieved using the incoherent model given by (4). For both cases, $\epsilon_{i}^{\prime \prime}$ was found to be $1.14 \times 10^{-2}$. Some slight adjustment of the thickness $d$ was required to achieve this agreement. At $35 \mathrm{GHz}$, we found that the angular behavior of the emission was better approximated by a coherent emission model as given in [4]. In this case we calculated $\epsilon_{i}^{\prime \prime}$ to be $5.6 \times 10^{-3}$.

In the aforementioned study perfomed by Mätzler and Wegmüller, their measurements of $\epsilon_{i}^{\prime \prime}$ cover several frequencies extending from 2.4 $\mathrm{GHz}$ to $94 \mathrm{GHz}$, for both pure and impure ice. Table 1 provides a com- 


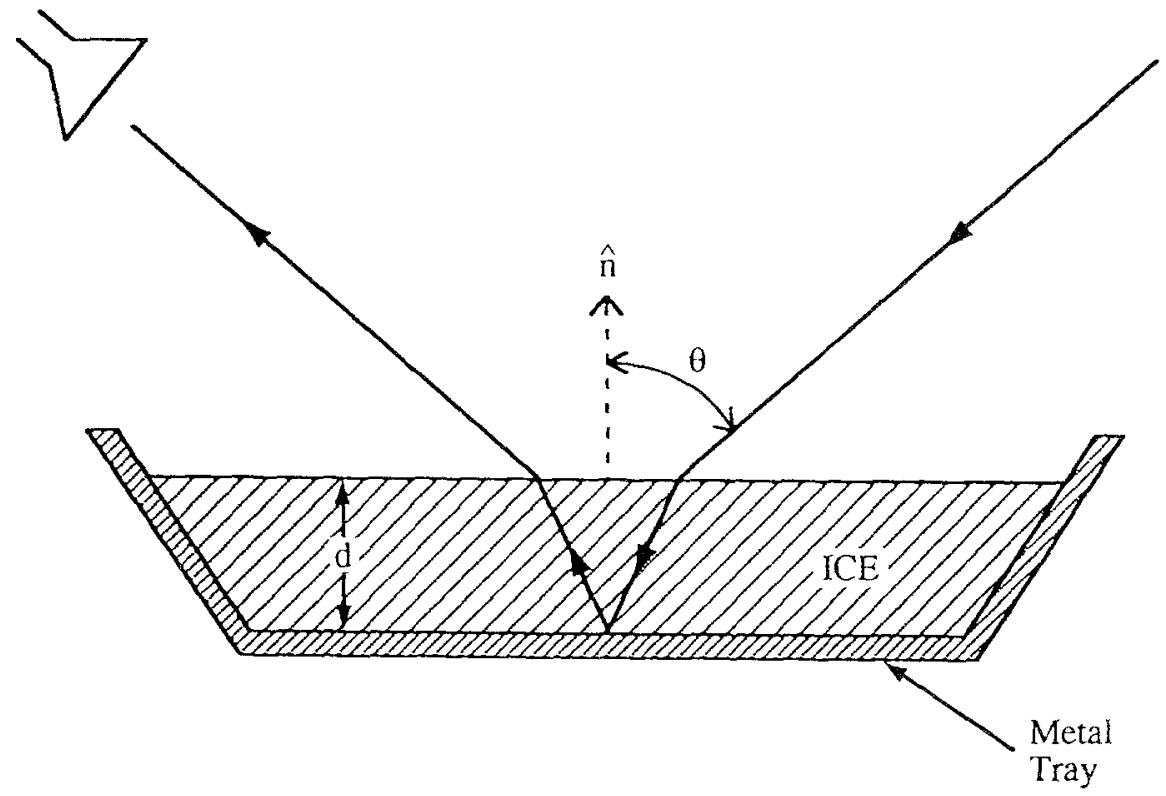

Figure 7: Schematic of experimental setup used to measure the radiometric temperature of ice. The results of such experiments were used to calculate the dielectic constant of ice. 


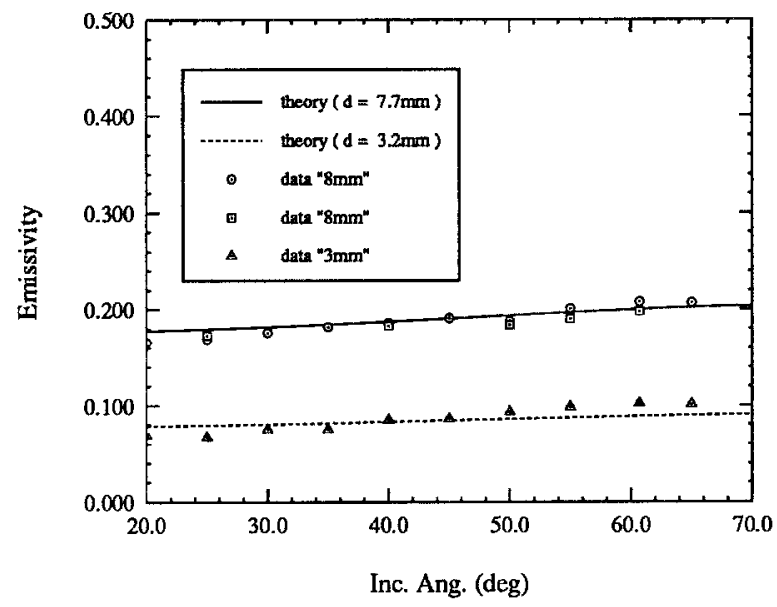

Figure 8: Experimental results of ice $\left(T_{\text {phys }}=-20^{\circ} \mathrm{C}\right)$ layer measurements at $94 \mathrm{GHz}$. for $\mathrm{V}$-polarization. Curves fitted to data were generated using an incoherent-emission model with $\epsilon_{I C E}=3.15-$ $\jmath 1.14 \times 10^{-2}$.

parison of their data for impure ice with our experimentally determined values of $\epsilon_{i}^{\prime \prime}$. The two sets of measurements are in close agreement, particularly at $94 \mathrm{GHz}$.

\begin{tabular}{|c|c|c|}
\hline Freq. (GHz) & $\begin{array}{c}\text { Previously } \\
\text { Reported }^{*}\end{array}$ & $\begin{array}{c}\text { This } \\
\text { Study }\end{array}$ \\
\hline 35 & $4.6 \times 10^{-3}$ & $5.6 \times 10^{-3}$ \\
\hline 94 & $1.13 \times 10^{-2}$ & $1.14 \times 10^{-2}$ \\
\hline
\end{tabular}

${ }^{*}$ Mätzler and Wegmüller, 1987.

Table 1: Experimentally determined values of $\epsilon_{i}^{\prime \prime}$ for impure ice. 


\section{Emission Model For Ice Layer Over SOFI Panel}

The values obtained for the dielectric constant of the SOFI panel and of the ice are the critical parameters in the final model which will predict emissivity as a function of ice thickness on the SOFI panel. Additionally, except possibly for thin ice layers examined at $35 \mathrm{GHz}$, our results show that the materials separately exhibit emission characteristics corresponding to incoherent processes. Since our measurements will principally involve the $94 \mathrm{GHz}$ channel, it is therefore appropriate to use incoherent radiative transfer theory to model the emission of the ice-SOFI structure.

\subsection{Isothermal Case}

Expressions were derived for the brightness temperature $T_{B}$ of an ice (or frost) layer of dielectric constant $\epsilon_{1}$ and thickness $d_{1}$ over a SOFI layer of dieletric constant $\epsilon_{2}$ and thickness $d_{s}=d_{2}-d_{1}$ [5]. The final expression for the emission from the uniform temperature case is given by:

$$
e=\left(1-R_{0}\right)\left[\frac{\left(1-R_{1} \Upsilon_{2}^{2}\right)-R_{1} \Upsilon_{1}^{2}\left(1-\Upsilon_{2}^{2}\right)-\Upsilon_{1}^{2} \Upsilon_{2}^{2}\left(1-R_{1}\right)}{\left(1-R_{1} \Upsilon_{2}^{2}\right)\left(1-R_{0} R_{1} \Upsilon_{1}^{2}\right)-\Upsilon_{1}^{2} \Upsilon_{2}^{2} R_{0}\left(1-R_{1}\right)^{2}}\right] T_{0}
$$

where

$$
\begin{aligned}
\Upsilon_{1} & =\exp \left(-\kappa_{1} d_{1} \sec \theta_{1}\right), \\
\Upsilon_{2} & =\exp \left[-\kappa_{2}\left(d_{2}-d_{1}\right) \sec \theta_{2}\right] \\
\kappa_{1} & =\frac{2 \pi}{\lambda} \frac{\epsilon_{1}^{\prime \prime}}{\sqrt{\epsilon_{1}^{\prime}}} \\
\kappa_{2} & =\frac{2 \pi}{\lambda} \frac{\epsilon_{2}^{\prime \prime}}{\sqrt{\epsilon_{2}^{\prime}}} \\
\theta_{1} & =\sin ^{-1}\left(\sin \theta_{o} / \sqrt{\epsilon_{1}^{\prime}}\right), \\
\theta_{2} & =\sin ^{-1}\left(\sin \theta_{o} / \sqrt{\epsilon_{2}^{\prime}}\right),
\end{aligned}
$$

and where $R_{0}$ and $R_{1}$ are the Fresnel reflectivities of the air/ice and ice/SOFI interfaces, respectively. 


\subsection{Non-Isothermal Case}

For the non-isothermal case, having a linear temperature gradient between the metal surface and the ice/SOFI interface (but constant throughout the ice layer) as shown in Figure 3, we compute not the emissivity but the brightness temperature representing the emission by the surface. This quantity is given by the following expression:

$$
\begin{aligned}
T_{B}= & \left\{\frac{\left(1-R_{0}\right)}{\left[\left(1-\Upsilon_{2}^{2} R_{1}\right)\left(1-\Upsilon_{1}^{2} R_{0} R_{1}\right)-\Upsilon_{1}^{2} \Upsilon_{2}^{2} R_{0}\left(1-R_{1}\right)^{2}\right]}\right\} \\
\times & \left\{T_{0}\left\{\left(1-\Upsilon_{1}\right)\left[\left(1+\Upsilon_{1} R_{1}\right)\left(1-\Upsilon_{2}^{2} R_{1}\right)+\Upsilon_{1} \Upsilon_{2}^{2}\left(1-R_{1}\right)^{2}\right]\right\}\right. \\
& \left.\quad+\Upsilon_{1}\left(1-R_{1}\right)\left(1-\Upsilon_{2}\right)\left(T_{1}+\delta+T_{0} \Upsilon_{2}\right)\right\}
\end{aligned}
$$

where all quantities are as defined above for the isothermal case and, additionally,

$$
\begin{aligned}
\delta & =\frac{\left(T_{0}-T_{1}\right)}{\tau_{s}}\left[\left(\tau_{s}-1\right)+\Upsilon_{2}\right] \\
\tau_{s} & =\kappa_{2} \sec \theta_{2}\left(d_{2}-d_{1}\right) .
\end{aligned}
$$

\section{$5 \quad$ Results}

The model proposed in Section 4 indicates that the $140 \mathrm{GHz}$ channel would be the most sensitive to small changes in ice thickness, thus providing the best resolution. Unfortunately, after obtaining only a small amount of data on the rough-surface SOFI panel, the $140 \mathrm{GHz}$ channel suffered a serious hardware failure and was not useable for the remainder of this study. Consequently, the most complete data sets we have were collected using the $94 \mathrm{GHz}$ channel.

\subsection{Smooth-Surface Panel-Isothermal Case}

Results for ice over the smooth-surface SOFI panel are shown in Figure 9. The experiment involved examining, using only vertical polarization, four separate thicknesses of ice over the SOFI panel: $2,4,6$, and $8 \mathrm{~mm}$. For each ice layer, the emissivity of the ice/SOFI system was measured at three different incidence angles. In the figure, The results of the experiment are presented along with the theoretical curves generated 


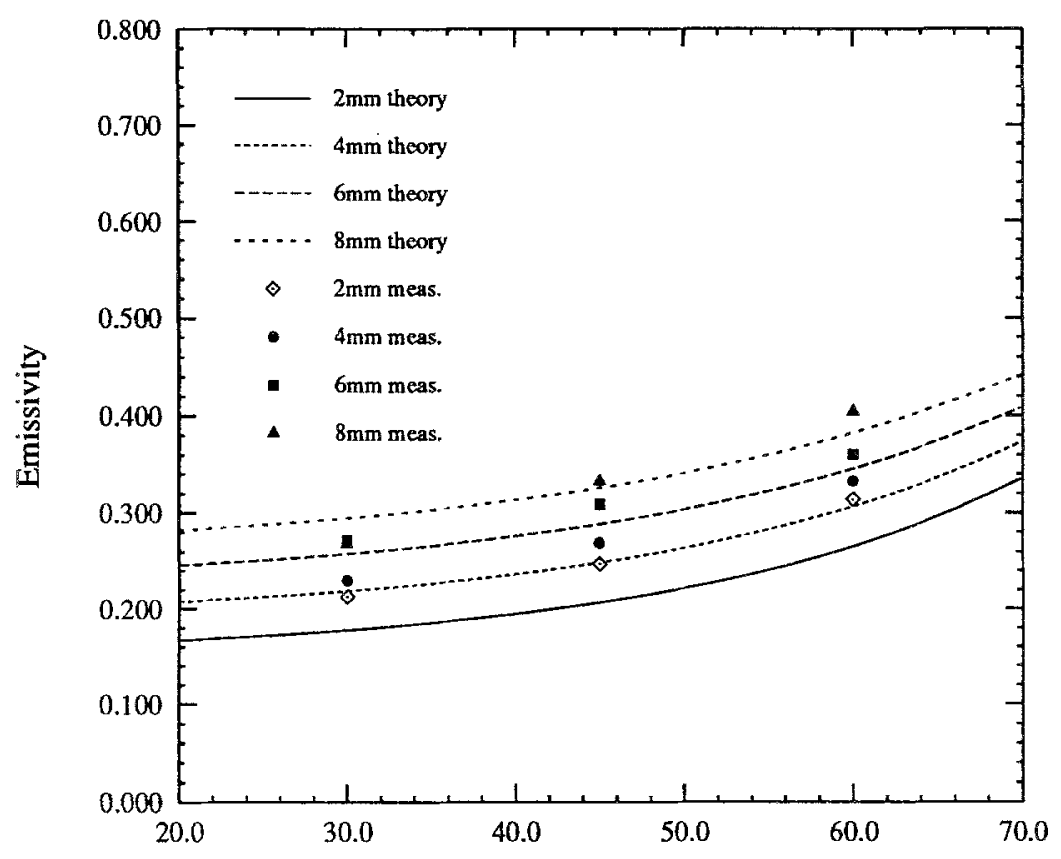

Inc. Ang. (deg)

Figure 9: Measured and predicted emissivity, at $94 \mathrm{GHz}$, for smoothsurface SOFI panel for four different ice thicknesses.

using the model (isothermal case) given in Section 4. This model uses the experimentally determined dielectric constants for the SOFI panel and for the ice, given in Sections 2 and 3, respectively. The agreement between the model and the actual measurements demonstrates that the behavior of a system of ice over SOFI panel can be quite accurately predicted using an incoherent radiative transfer model, if the top surface of the SOFI panel is smooth.

For this case, the sensitivity of the method, that is, the change in emissivity with respect to change in ice thickness is illustrated in Figure 10. Here is shown, for a particular incidence angle of $30^{\circ}$, the variation of the emissivity with ice thickness at 35,94 , and $140 \mathrm{GHz}$ for the case of pure ice (Figure 10a) and for the case of frost-like ice, having 
an ice volume density $v$ of 0.5 (Figure 10b). Actual data is shown for $94 \mathrm{GHz}$ for the pure ice case; the curves for $35 \mathrm{GHz}$ are based on our experimentally determined value of $\epsilon_{i}^{\prime \prime}$; and at $140 \mathrm{GHz}$ we have used a value based on extrapolating the data of Mätzler and Wegmüller [3] for impure ice to $140 \mathrm{GHz}$.

Figure 11 presents essentially the same information, except that now we show the variation of antenna temperature $T_{A}$ with ice thickness, which includes the sky reflected contribution (see (1)). In this case, it is necessary to assume a representative value of $T_{S K Y}$ for each of the three frequencies. The resulting values for $T_{A}$ indicate that for pure ice, the antenna temperature exhibits an approximately linear variation with ice thickness, with a slope of $5.3 \mathrm{~K} / \mathrm{mm}$ at $140 \mathrm{GHz}, 4.2 \mathrm{~K} / \mathrm{mm}$ at 94 $\mathrm{GHz}$, and $1.2 \mathrm{~K} / \mathrm{mm}$ at $35 \mathrm{GHz}$.

\subsection{Smooth-Surface Panel with Temperature Gra- dient}

In the isothermal case treated in the preceeding section, the ice thickness sensing process would involve two steps:

1. The emissivity $e$ is obtained from (3), repeated here for clarity,

$$
e=\frac{T_{A}-T_{S K Y}}{T_{p h y s}-T_{S K Y}}
$$

by measuring $T_{A}$ and $T_{S K Y}$ with the radiometer and $T_{\text {phys }}$ with a contact sensor or an infrared radiometer.

2. The emissivity is related to ice thickness $d$ by using the curves given in Figure 10.

As alluded to in Section 1, the fueltank/ice system is in reality not an isothermal system. It is more accurately described in terms of a temperature gradient, having a temperature on the order of $94 \mathrm{~K}$ at the metal-SOFI interface, and warming up to a temperature just below the freezing point of water, about $270 \mathrm{~K}$, at the SOFI-ice interface [2]. Hence in this case we cannot use (3) in its present form because $T_{p h y s}$ no longer has a unique value. However, we can develop an expression similar in form to (3) for the temperature-gradient situation by: 


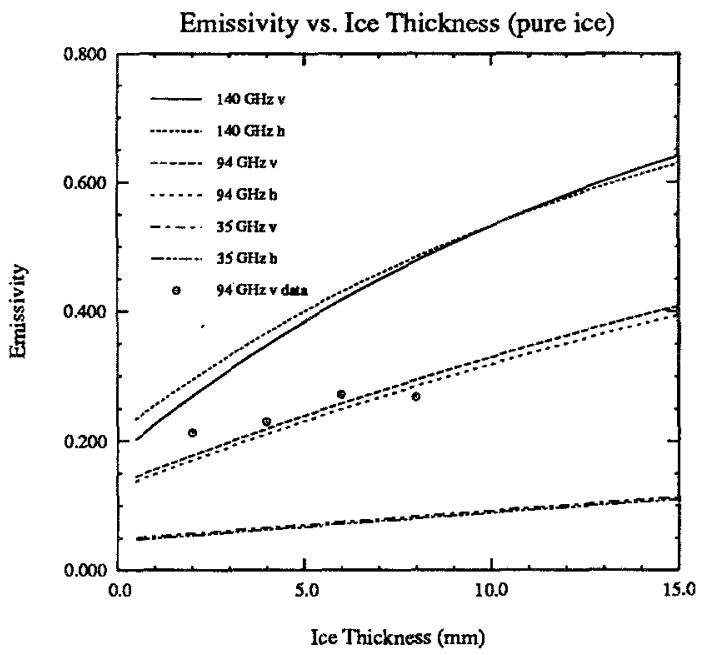

(a)

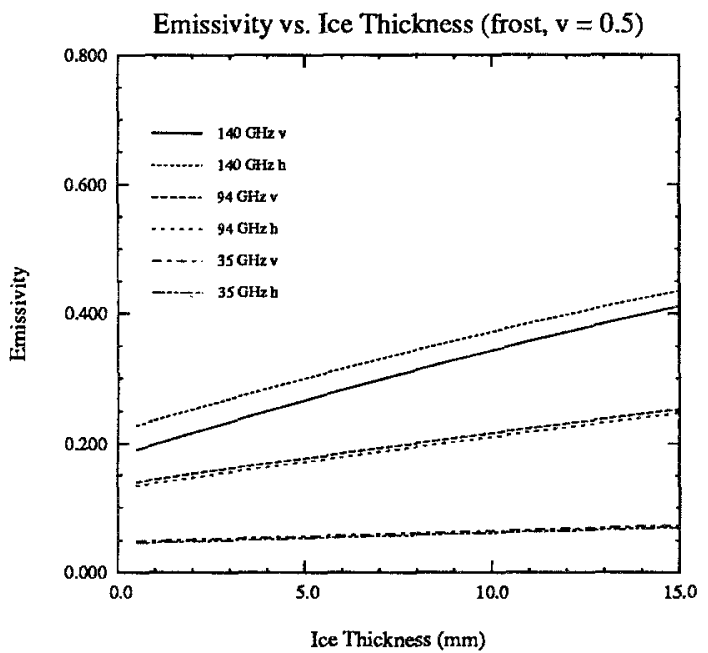

(b)

Figure 10: Isothermal case: Emissivity variation with ice thickness for ice volume density of (a) 1.0 (ice) and (b) 0.5 (frost). 


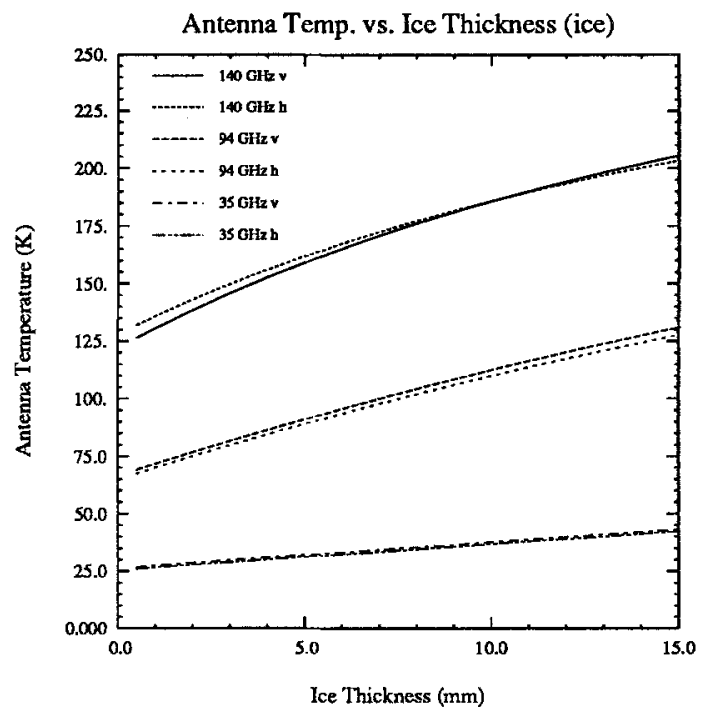

(a)

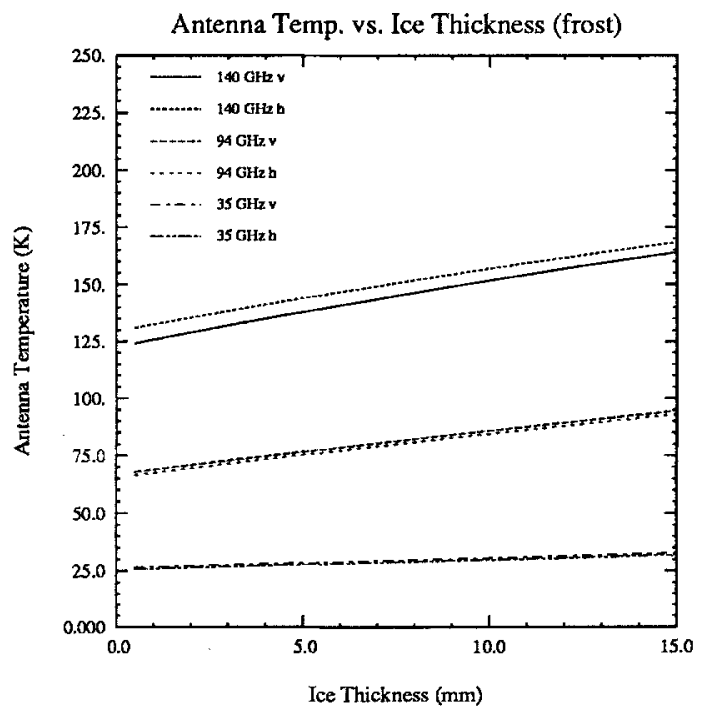

(b)

Figure 11: Isothermal case: Antenna temperature variation with ice thickness for ice volume density of (a) 1.0 (ice) and (b) 0.5 (frost). The model calculations are for $T_{P H Y S}=270 \mathrm{~K}$ and $T_{S K Y}=14,35$, and $90 \mathrm{~K}$ for 35,94 , and $140 \mathrm{GHz}$ respectively. 


\begin{tabular}{|r|c|c||c|c|||c|c||c|c|}
\cline { 2 - 9 } \multicolumn{1}{c|}{} & \multicolumn{4}{c||}{ Vertical Polarization } & \multicolumn{3}{c|}{ Horizontal Polarization } \\
\cline { 2 - 9 } \multicolumn{1}{c|}{} & Frost $(\mathrm{v}=0.5)$ & \multicolumn{1}{c||}{ Ice $(\mathrm{v}=1.0)$} & Frost $(\mathrm{v}=0.5)$ & \multicolumn{1}{c|}{ Ice $(\mathrm{v}=1.0)$} \\
\hline Freq. (GHz) & $\mathrm{m}$ & $\mathrm{b}$ & $\mathrm{m}$ & $\mathrm{b}$ & $\mathrm{m}$ & $\mathrm{b}$ & $\mathrm{m}$ & $\mathrm{b}$ \\
\hline 35 & 0.990 & 0.984 & 0.993 & 0.984 & 0.985 & 0.985 & 0.994 & 0.985 \\
\hline 94 & 0.971 & 0.953 & 0.971 & 0.953 & 0.974 & 0.955 & 0.968 & 0.956 \\
\hline 140 & 0.962 & 0.936 & 0.954 & 0.935 & 0.920 & 0.948 & 0.938 & 0.921 \\
\hline
\end{tabular}

Table 2: Values for slope and intercept which relate the reflectivity to the effective emissivity for a ice/SOFI system having a linear temperature profile. These values are for the case of a $30^{\circ}$ incidence angle.

1. defining $e_{e f f}$ as the effective emissivity given by

$$
e_{e f f}=\frac{T_{B}}{T_{0}}
$$

where $T_{B}$ is the brightness temperature and $T_{0}$ is the physical temperature of the medium at its upper surface (in our case, $T_{0}$ is the ice temperature, typically $270 \mathrm{~K}$ ) and,

2. relating $e_{e f f}$ to the reflectivity $R$ using the linear form

$$
R=b-m e_{e f f}
$$

Upon inserting (13) and (14) into (1) and solving for $e_{e f f}$, we obtain the result

$$
e_{e f f}=\frac{T_{A}-b T_{S K Y}}{T_{0}-m T_{S K Y}}
$$

This procedure is predicated upon the assumption that $e_{e f f}$ and $R$ are linearly related as indicated by (14). This was shown to be indeed the case through the use of (12) to compute $T_{B}$ and (11) to compute $R=1-e$. Note that this reflectivity computed for the isothermal case is equally valid for the temperature gradient case since it is a property of the material itself, not of the temperature profile of the material. After computing $e_{e f f}$ and $R$ for many values of ice thickness $d$, regression analysis was used to determine the constants $b$ and $m$ in (14). Table 2 provides a summary of the results for pure ice and for frost at 35, 94, and $140 \mathrm{GHz}$. For all cases the multiple correlation coefficient $r^{2}$ was greater than 0.99 . 


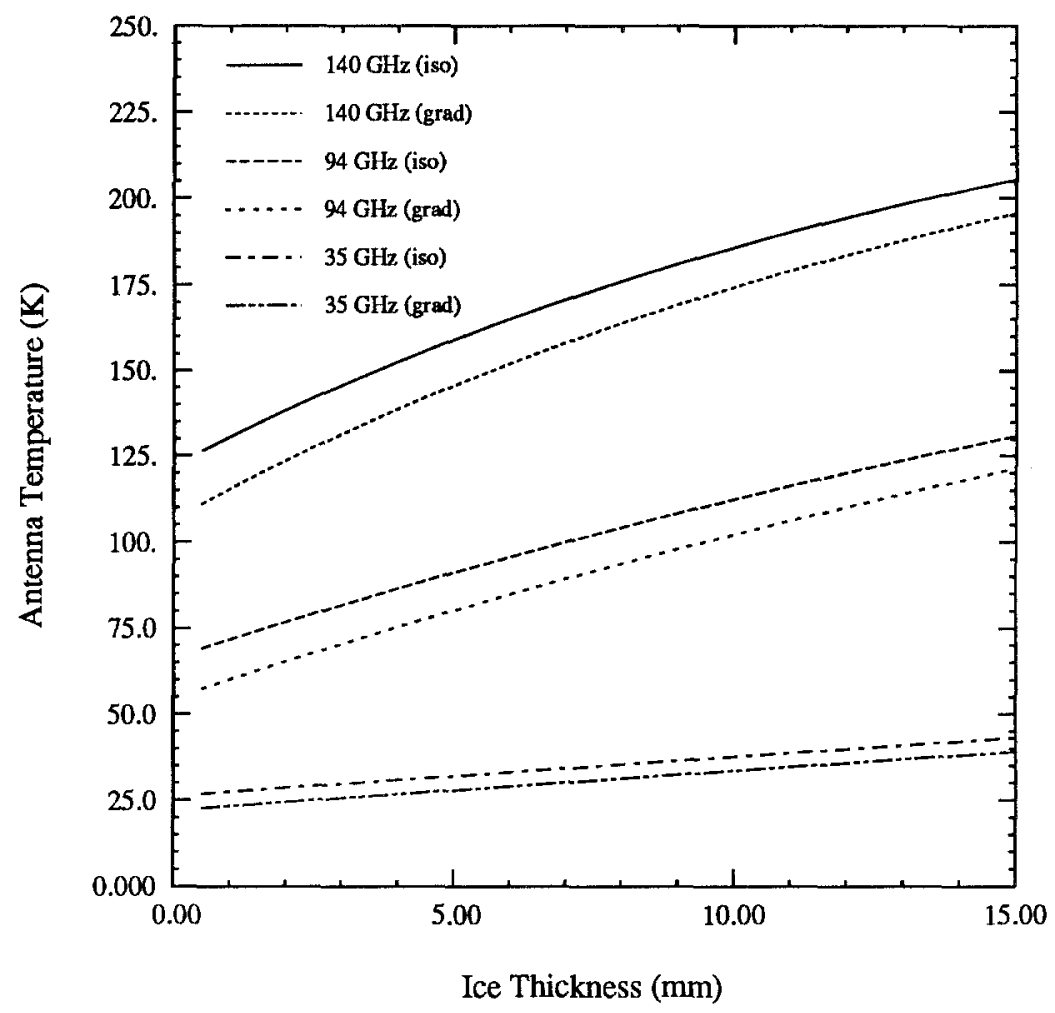

Figure 12: Antenna Temperature versus ice thickness for isothermal and temperature-gradient cases (with $T_{P H Y S}=270 K$ and $T_{S K Y}=14$, 35 , and $90 \mathrm{~K}$ for 35,94 , and $140 \mathrm{GHz}$ respectively).

Figure 12 compares the variation of antenna temperature with ice thickness for the SOFI/ice system for both the isothermal case and the temperature gradient case. The antenna temperature $T_{A}$ is somewhat lower for the temperature-gradient case but its sensitivity to ice thickness is slightly greater. The latter point is summarized in Table 3 for the pure $(\mathrm{v}=1.0)$ ice case.

One implication of these differences is that the accuracy of icethickness determination is impaired if isothermal-based calculations are used for a system having a temperature gradient. If such an approximation were feasible, the need for determining empirical constants $m$ and $b$ described in this section could be avoided. From Figure 12, in the 


\begin{tabular}{|r|c|c|}
\multicolumn{1}{c|}{} & \multicolumn{2}{c|}{ Sensitivity $(\mathrm{K} / \mathrm{mm})$} \\
\cline { 2 - 3 } \multicolumn{1}{c|}{} & Temperature Profile \\
\hline Freq. $(\mathrm{GHz})$ & Uniform & Linear \\
\hline 35 & 1.2 & 1.3 \\
\hline 94 & 4.2 & 4.5 \\
\hline 140 & 5.0 & 5.7 \\
\hline
\end{tabular}

Table 3: Sensitivity, in $\mathrm{K} / \mathrm{mm}$, of antenna temperature to changes in ice thickness. Values are for an incidence angle of $30^{\circ}$. Results shown are valid for both polarizations.

case of pure ice, it can be seen that such an attempt would consistently underestimate the ice thickness by about $2.5 \mathrm{~mm}$ for the $94 \mathrm{GHz}$ case, and $1.5-2.0 \mathrm{~mm}$ for the $140 \mathrm{GHz}$ case.

One difficulty with the temperature gradient case is that the exact magnitude of the temperature gradient might not be known. While the surface temperature of the ice/SOFI system can be relatively easily determined, there would likely be more uncertainty in knowing the temperature at the metal/SOFI interface. A logical question is how seriously would incorrect descriptions of the temperature gradient affect the accuracy of the ice thickness estimations. Our investigations have shown that $T_{A}$ is actually quite insensitive to variations of this kind and that the temperature at the metal/SOFI interface could differ from 94 $\mathrm{K}$ by $80 \mathrm{~K}$ and induce an ice thickness estimation error of only $1 \mathrm{~mm}$.

\subsection{Rough-Surface Panel}

The experiments performed with the rough-surface (original) SOFI panel were identical to those described above for the smooth-surface panel; that is, four different ice thicknesses were observed with a vertically polarized radiometer. The results indicate that the rough surface significantly modifies the emission from the ice-SOFI structure relative to what the model predicts and what was observed for the smoothsurface system. The antenna temperature $T_{A}$ was on the average some $65 \mathrm{~K}$ higher than observed for the corresponding smooth-surface case. The rough surface was unimportant when observations were made of the SOFI panel alone because of the very small dielectric contrast between the SOFI material and air. When ice-covered however, it becomes a rough interface of significant dielectric contrast. 
An attempt was made to model the emission problem by describing the ice/SOFI interface as a rough surface using the stationary-phase approximation of the Kirchoff approach [6]. We succeeded in reproducing qualitatively the increase in the level of the apparent temperature relative to that of the smooth-surface case, but it was not identical to the experimental rough-surface results, and more seriously, it exhibited a different dependence with respect to ice thickness $\left(\delta T_{A} / \delta d_{i c e}\right)$.

Although strictly speaking the relation $e=1-R$ may not be valid when the ice/SOFI interface is rough, we will nevertheless use it as a first-order approximation in order to compare the emissivity results for the rough-surface boundary with those for the smooth-surface boundary. As depicted in Figure 13, both the angular variation (13a) and the sensitivity with respect to ice thickness (13b) of the emissivity for the rough-surface case closely resemble the corresponding properties for the smooth-surface case. In short, it appears as though the rough ice/SOFI interface has the effect of imparting a bias (on the order of $65 \mathrm{~K}$ ) to $T_{A}$ (relative to the smooth-surface system), but otherwise preserves the essential emission behavior seen in the smooth-surface case. A model which can account for such behavior remains the subject of future research.

\section{Conclusion}

The purpose of this investigation has been to determine whether it is feasible to sense the presence and thickness of ice on the surface of the Space Shuttle fueltank using a radiometer operating at millimeter wave frequencies. More specifically, we have attempted to determine whether intrinsic material quantities such as emissivity and reflectivity, derived from radiometric measurements, could be used to predict ice thickness.

For the case in which the normally rough fueltank material (SOFI) has been made smooth, we have demonstrated that a model based on radiative transfer theory can correctly predict emissivity for a given ice thickness. We have verified this model with experiments performed on a system of ice/SOFI in an isothermal state, and have shown how the technique can be extended to the more realistic case of a temperature gradient.

For the case of the SOFI material in its original (rough-surface) state, we have observed that the antenna temperature is modified sig- 
nificantly beyond what is predicted by the smooth-surface model described above. We have shown some evidence that suggests that the sole effect of the rough ice/SOFI interface is to impart a bias on the order of $65 \mathrm{~K}$ to $T_{A}$ relative to the smooth-surface case. A model which can account for the behavior of such a system remains the subject of further investigation. 


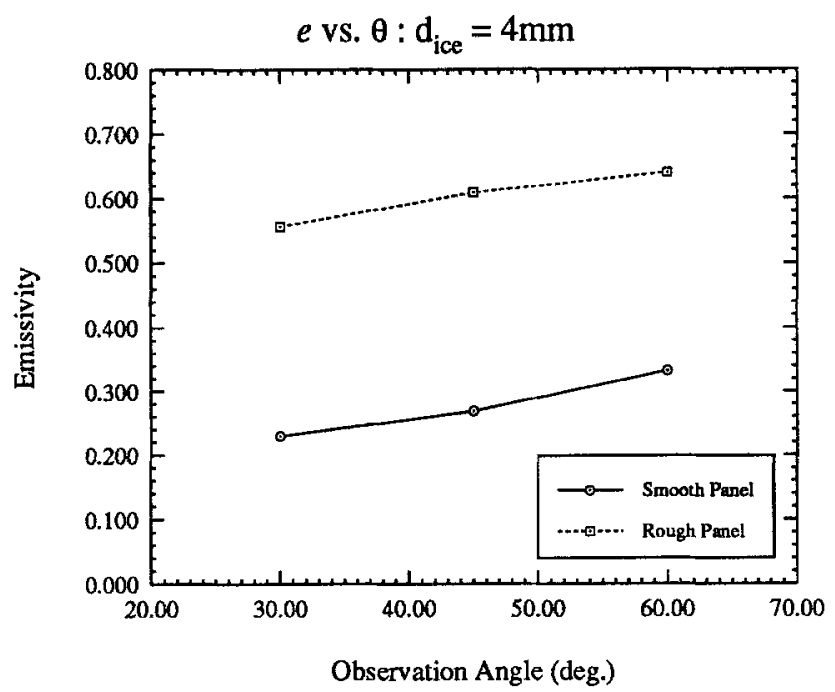

(a)

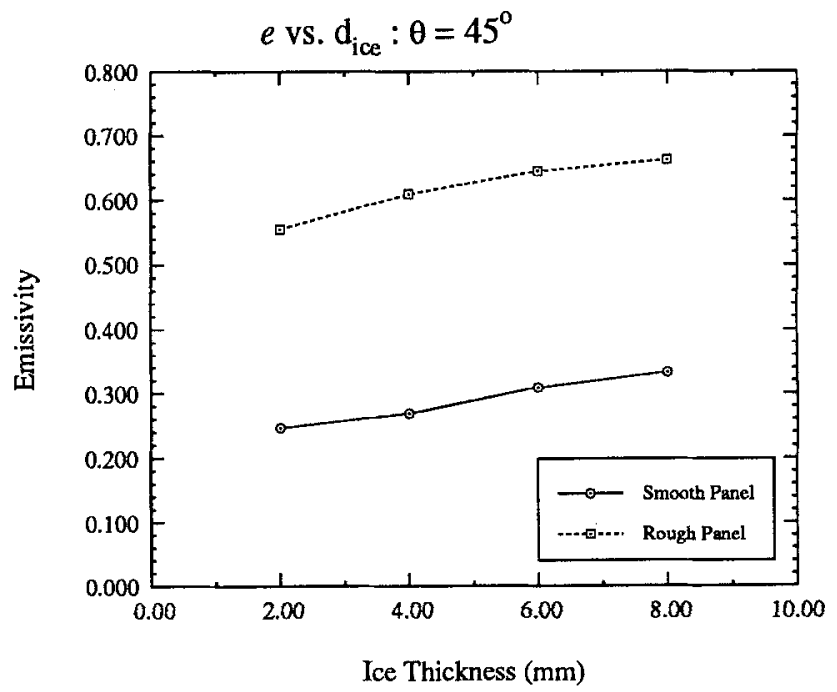

(b)

Figure 13: Comparison of measured emissivity at $94 \mathrm{GHz}$ for the roughsurface (original) SOFI panel with that for the smooth-surface SOFI panel: Dependence of emissivity on (a) incidence angle, and (b) ice thickness. 


\section{References}

[1] Ulaby, F.T., R.K. Moore, and A.K. Fung, Microwave Remote Sensing, vol.3, Artech House, Dedham, Mass., 1986.

[2] Wu, S., NASA Stennis Research Center, personal communication.

[3] Mätzler, C., and U. Wegmüller, "Dielectric Properties of Fresh Water Ice at Microwave Frequencies," J. Phys. D: Applied Physics, vol. 20, 1987, pp. 1623-1630.

[4] Ulaby, F.T., R.K. Moore, and A.K. Fung, Microwave Remote Sensing, vol.1, Addison-Wesley, Reading, Mass., Ch. 4, 1981.

[5] Ulaby, F.T., J.R. Kendra, T. Haddock, and S. Wu, "A MillimeterWave Radiometer Technique for Measuring Ice Thickness on the Surface of the Space Shuttle's External Fueltank," Radiation Lab Report 027595-F, The University of Michigan, February, 1991.

[6] Ulaby, F.T., R.K. Moore, and A.K. Fung, Microwave Remote Sensing, vol.2, Addison-Wesley, Reading, Mass., Ch. 12, 1982. 